\title{
Segmentation of the Pectoral Muscle in Breast MRI Using Atlas-Based Approaches
}

\author{
Albert Gubern-Mérida ${ }^{1}$, Michiel Kallenberg ${ }^{2}$, \\ Robert Martí ${ }^{1}$, and Nico Karssemeijer ${ }^{2}$ \\ 1 University of Girona, Spain \\ \{agubern, marly\}@eia.udg.edu \\ 2 Radboud University Nijmegen Medical Centre, The Netherlands \\ $\{$ m.kallenberg,n.karssemeijer\}@rad.umcn.nl
}

\begin{abstract}
Pectoral muscle segmentation is an important step in automatic breast image analysis methods and crucial for multi-modal image registration. In breast MRI, accurate delineation of the pectoral is important for volumetric breast density estimation and for pharmacokinetic analysis of dynamic contrast enhancement. In this paper we propose and study the performance of atlas-based segmentation methods evaluating two fully automatic breast MRI dedicated strategies on a set of 27 manually segmented MR volumes. One uses a probabilistic model and the other is a multi-atlas registration based approach. The multi-atlas approach performed slightly better, with an average Dice coefficient (DSC) of 0.74 , while with the much faster probabilistic method a DSC of 0.72 was obtained.
\end{abstract}

Keywords: pectoral muscle, breast MRI, atlas-based segmentation.

\section{Introduction}

Automatic identification of pectoral muscle is an important step in methods for automatic breast cancer assessment in most image modalities. For instance, in mammography, the most used image modality in screening programs, the detection and removal of the pectoral muscle is often used to remove false positive marks of Computer Aided Detection (CAD) systems [3. In Magnetic Resonance Imaging (MRI) of the breast, the image modality employed in the presented work, the importance of the pectoral muscle detection has recently been recognized in two applications. Firstly, breast tissue density has been identified as an important risk factor for developing breast cancer, being four times larger in women with a breast density higher than $75 \%$, compared to those with little or no density 2]. Breast MRI provides a good tissue contrast between fibroglandular and fatty tissues and a three-dimensional characterization of breast composition. These good properties in the breast tissue have been a strong reason to use breast MRI in breast density measurement 998,5$]$. However, the contrast between pectoral muscle and dense tissue is poor. Hence, a first step to separate the breast from the body is commonly essential. This separation is not trivial 
due to the large shape and intensity variations in the pectoral muscle of different patients. Some solutions are present in the literature: boundary tracing or spline fitting without [10] and with manual intervention [98] and delineation of the whole breast using breast models 4, but none of them completely delineates the pectoral muscle.

Secondly, MRI is often used with a contrast agent for lesion detection. For a better interpretation of contrast enhancement lesions, researchers have tried to incorporate pharmacokinetic modeling to the interpretation of the MRI. Some of these models require calibrations with respect to reference tissues and make use of the signal intensity of specific regions for determining physiological measures [11. In breast MRI, the pectoral muscle can be used as a reference tissue given its properties.

Atlas-based segmentation has been shown to be a powerful technique for automatic delineation of anatomical structures in different 3D image modalities [16]. Multi-atlas and probabilistic approaches are the most commonly used strategies. By definition, the former is supposed to obtain more precise segmentations than the latter. However, multi-atlas approach is far more time consuming. There has been only one initial attempt that uses an atlas strategy for breast MRI segmentation [5], but the segmentation of the pectoral muscle was not the main interest of the work. Moreover, the method followed a probabilistic approach using one reference atlas, which could have some limitations. As shapes are highly variable, the reference choice affects final results.

The novelty of this paper consist in the study of fully automatic atlas-based methods for pectoral muscle segmentation in breast MRI in terms of performance and complexity. A dedicated multi-atlas approach based on [6] is proposed (see section [3.3) and compared to the probabilistic approach of [5] (see section [3.2). An original breast MRI registration framework focused on the body has been also defined and used in both methods (see section 3.1). To our knowledge, no similar studies are found in the literature. Advantages and inconveniences of both strategies are discussed in sections 4 and 5 and a solution to obtain a reasonable time-accuracy trade-off is proposed.

\section{Material}

The data set used to evaluate the segmentation results and build the atlases consists of 27 pre-contrast T1-weighted MR breast scans obtained from different patients. Breast MRI examinations were performed on a $1.5 \mathrm{~T}$ system (Siemens 1.5T, Magnetom Vision), with a dedicated breast coil (CP Breast Array, Siemens, Erlangen). The pixel spacing differed between volumes with values ranging from $0.625 \mathrm{~mm}$ to $0.722 \mathrm{~mm}$. The slice thickness was $1.3 \mathrm{~mm}$ and the volume size was 512 x 256 x 120 voxels. Patients were scanned in prone position.

Three experienced observers performed manual segmentations. Two of them manually segmented only the pectoral muscles of 8 cases. The third one manually segmented each of the $27 \mathrm{MR}$ volumes into 7 classes: background, fatty tissue, glandular tissue, pectoral muscles, lung area and the heart. The seventh class is the "other" class and refers the previous non-labeled voxels of the thorax. 
Annotations were done every 5-10 slices and linear interpolation was applied to obtain the complete labeling. When needed, and especially for heart, lungs and pectoral muscles, accurate manual delineation was performed with a smaller slice interval. For the manual segmentation of background, fatty and fibroglandular tissue, thresholding was applied over regions of interest provided by the reader. Fig. 11 shows an example of a MRI slice on an axial view and the manual delineation of the mentioned classes. One should note the complexity of performing such ground truth annotations, where each volume takes approximately 45 minutes in a dedicated breast MRI annotation environment.

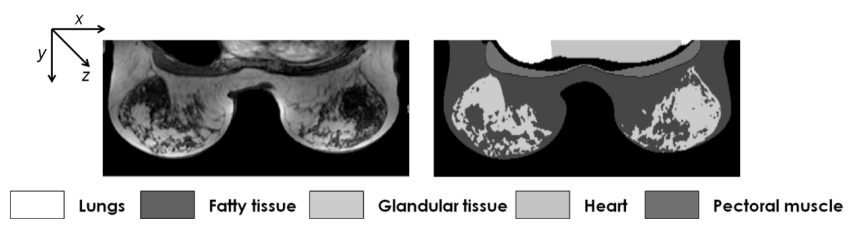

Fig. 1. Breast MR scan on an axial slice with the manual annotation of the different structures

\section{Methods}

Atlas-based strategies are characterized by the use of prelabeled images, usually manually obtained, to perform the automatic segmentation of new images, also called targets. They employ registration algorithms, which play an important role for the final segmentation. Section 3.1 explains the mapping algorithm used by the two atlas-based approaches evaluated in this paper. Section 3.2 briefly describes the construction and the use of a probabilistic atlas in a Bayesian framework segmentation [5. Finally, in section 3.3 we report the proposed multiatlas segmentation algorithm based on [6] for the delineation of the pectoral muscle in breast MRI.

\subsection{Registration}

Registration is an important step in atlas-based segmentation algorithms. Without an accurate transformation between the structures we aim to segment, the segmentation can not perform accurately. For this reason we developed a registration framework focused on the body area. We observed that the sternum is always localized between pectoral muscles. Hence, by accurately localizing the sternum the pectoral muscles can be aligned. Our registration approach is initialized by detecting the sternum of the subjects. Automatic sternum detection is based on [5]. Then, the volumes are cropped at $2 \mathrm{~cm}$ distance anterior to the sternum position to focus the registration on the area of the body. By doing so, most breast tissue is removed and can not negatively bias the final mapping of body structures. The $2 \mathrm{~cm}$ distance anterior to the sternum ensures that pectoral muscle voxels are not discarded. 
The registration process is composed by two stages. First, a translation transform is performed, where translation along the $y$ axis is defined by the distance between $y$-coordinates of both sternums. Translation along $x$ and $z$ axis is found by optimizing the similarity measure. The second stage is a non-rigid transform based on B-Splines registration in a multi-resolution scheme using a stochastic gradient descent optimizer. Three resolutions were defined. B-Splines grid spacing was set to 32,16 and $8 \mathrm{~mm}$ for each of the resolutions taking the size of the pectoral muscle into account. The similarity measure maximized by the whole framework was normalized cross correlation (NCC) as all the datasets were acquired with the same modality. Elastix [7] was used for the implementation.

\subsection{Method 1: Probabilistic Atlas-Based Segmentation}

In the presented atlas-based segmentation method, a probabilistic atlas is used in a Bayesian framework to provide an accurate probability distribution for the pectoral an the thoracic area. Following a leave-one-out evaluation strategy, for each patient segmentation, a full probabilistic atlas was built offline with the 26 remaining patients. These 26 patients and their segmentations were mapped using the registration method explained previously into the same reference space. The probabilistic atlas was created by computing the frequency with which each location was labeled as a specific organ. A common reference space was used for all the experiments by visually selecting an extra patient which has normal appearance. The reference case, or anatomical image of the atlas, was not included in the evaluation set.

Figure 2 shows the general schema of the segmentation framework with Bayesian voxel classification algorithm incorporating the use of the probabilistic atlas. From the top to bottom, the probabilities of the atlas are mapped by registration of section 3.1 onto target image space $\{\mathrm{T}\}$ using the anatomical image

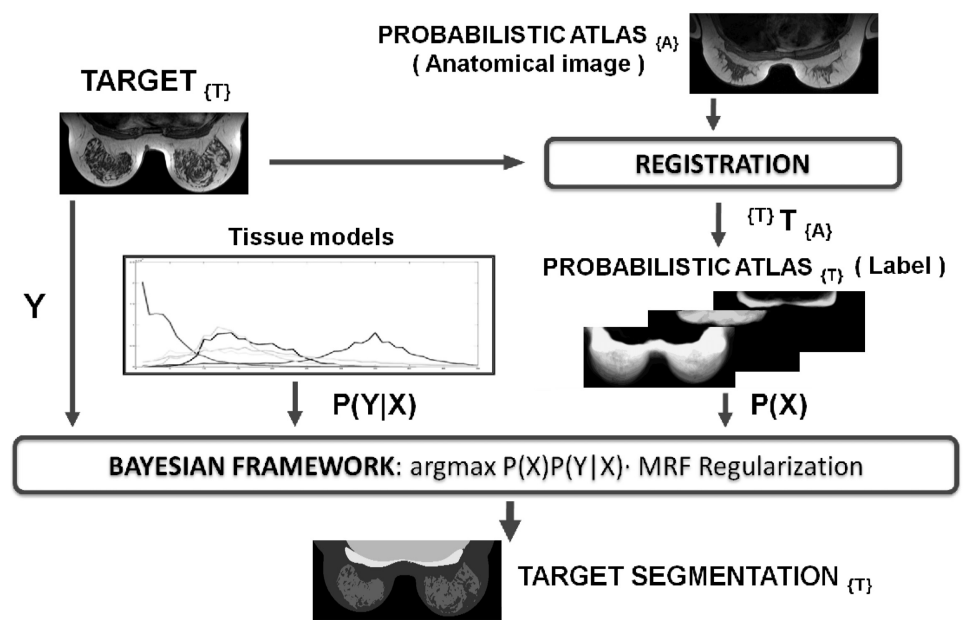

Fig. 2. Probabilistic atlas segmentation approach overview 
of the atlas. The probabilistic atlas, the tissue models (previously built from the scans and manual segmentations of the data set) and the target are provided to the Bayesian framework as a prior probability $P(X)$, conditional probability $P(Y \mid X)$ and set of intensity values $Y$, respectively. The Bayesian framework estimates the segmentation $X$ that maximizes $P(X) P(Y \mid X)$ and also includes a Markov Random Field (MRF) regularization to smooth the segmentation taking neighborhood information into account [5].

\subsection{Method 2: Multi-atlas Segmentation}

Multi-atlas segmentation approaches consider all the volumes of the dataset and their manual segmentations as individual atlases. The term atlas is defined as the pair of the anatomical image (MRI volume) and its manual segmentation or label. The process to obtain an automatic segmentation for a target volume is illustrated in figure 3. First, given the target volume $T$, all the atlases are mapped onto the target space using the registration algorithm of section 3.1 Subsequently, the deformed anatomical images are compared to the target to perform a selection of the most similar atlases. The selection is based on the Normalized Cross Correlation similarity measure and a ratio defined as follows:

$$
r_{i}=\frac{N C C\left(T, A_{i} \circ M_{i}\right)}{\max _{j} N C C\left(T, A_{j} \circ M_{j}\right)},
$$

where $M$ refers to the mapping between the target and an atlas and $j$ refers to the deformed atlas with maximum similarity. An atlas $A_{i}$ is selected if it satisfies $r_{i} \geq \varphi$. A value of $\varphi=0.9$ empirically appeared to be the best value for our results.

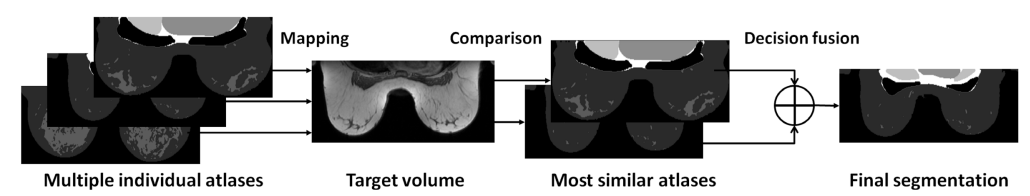

Fig. 3. Multi-atlas segmentation approach overview

Finally, the selected deformed atlas labels are fused to yield a single final segmentation of the patient or target image. This step is called decision fusion and defines how the deformed segmentations of the selected atlas are combined. In this work we have made use of majority voting method, which was proven to give good results in 6 .

\section{Results}

In a leave-one-out experiment we evaluated the probabilistic and the multi-atlas segmentation frameworks on 27 patients. Each segmented case was not included 
for the construction of the probabilistic atlas or within the set of individual atlases respectively. The quality of the segmentation was measured by determining the similarity of the segmentation with the ground truth using the Dice Similarity Coefficient (DSC). DSC was chosen as it is commonly used in the literature 6 65. For all cases we manually discarded initial and last slices which do no contain relevant information or are clearly affected by noise. Figure 4(a) shows a box plot with DSC values for each method. Segmentation results are similar (DSC median of 0.76 for both and DSC mean \pm sd of $0.72 \pm 0.09$ and $0.74 \pm 0.06$ for probabilistic and multi-atlas respectively), but multi-atlas framework slightly outperforms the probabilistic. These results can be better seen in figure 4(b), where DSC values of each case using both methods are shown.

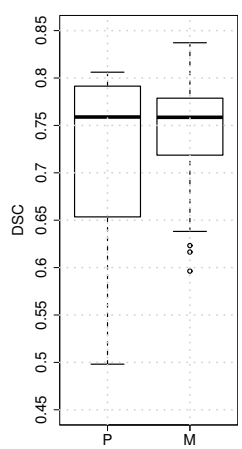

(a)

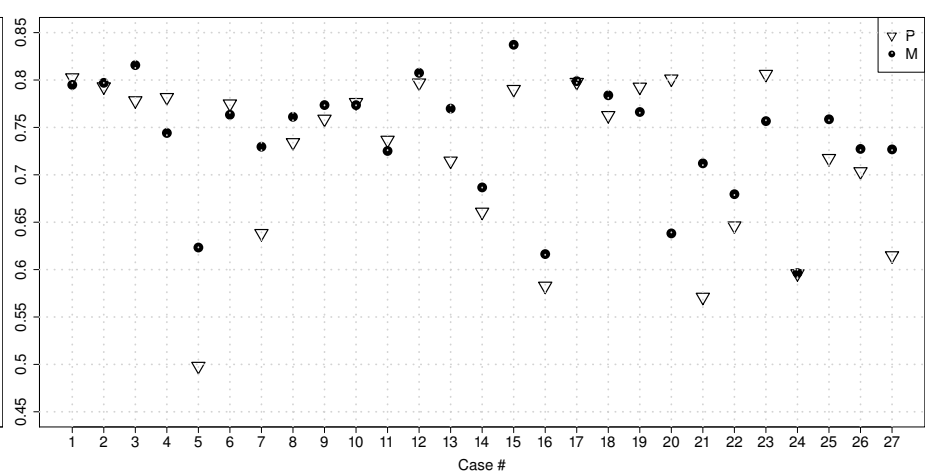

(b)

Fig. 4. (a) Box plot with segmentation DSC for pectoral segmentation and (b) DSC segmentation results for each of the 27 cases using probabilistic (P) and multi-atlas (M) approaches

Lower DSC values are mainly due to the registration process not being able to compensate the differences between volumes. This is more the case of the probabilistic approach as the method uses only one registration with a single reference. In those cases (see case 7 for instance), the multi-atlas approach performs better as it includes multiple registrations and selects the best ones. Only in one case (number 20) the probabilistic approach obtains results much better than multi-atlas, where pectoral muscle segmentation in initial slices is not really precise (labeled as thorax instead). However, accurate delineations in intermediate slices are obtained for both methods as it is illustrated in figure 5. where three examples of automatic and manual segmentations are shown.

Finally, since no previous works performed pectoral segmentation in breast MRI, inter-observer variability was computed by 3 viewers over 8 manual segmentations. DSC mean of $0.70 \pm 0.12$ and median of 0.72 were obtained, lower than the DSC values achieved by the automatic atlas-based approaches.

All the tests have been launched on Intel(R) Core(TM)2 Quad CPU Q9550 $2.83 \mathrm{GHz}$. Starting with the common step, registration between two volumes takes 


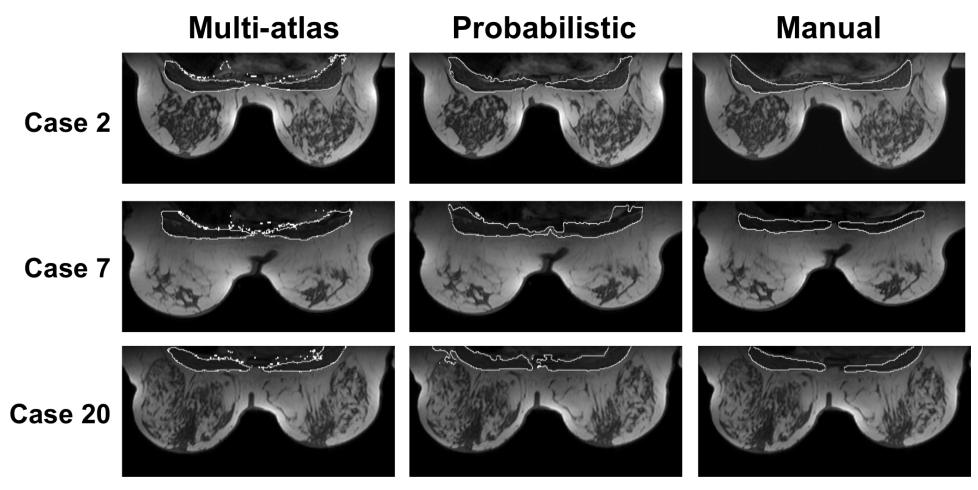

Fig. 5. Intermediate slices from 3 different cases and their segmentations

$t_{r} \approx 12 \mathrm{~min}$. The complexity time for multi-atlas segmentation is explained as $t_{\text {multi-atlas }} \approx N \times\left(t_{r}+t_{a}\right)+n \times t_{f}$, where $N$ is the number of individual atlases $(N=26), t_{a}$ the time to compute the mapping and the comparison of an individual anatomical atlas $\left(t_{a} \approx 4 \mathrm{~min}\right.$.), $n$ the number of selected atlases and $t_{f}$ the time to propagate and fuse an atlas labeled image $\left(t_{f} \approx 3 \mathrm{~min}\right.$.). In the best scenario, being only one atlas selected $(n=1)$, the computation time to obtain a segmentation using a multi-atlas approach is $t_{\text {multi-atlas }} \approx 419$ minutes ( 7 hours). The complexity time for the probabilistic approach is defined as $t_{\text {probabilistic }} \approx t_{r}+t_{p}+t_{b}$, where $t_{p}$ is the time to map the probability distributions to the target space $\left(t_{p} \approx 8 \mathrm{~min}\right)$ and $t_{b}$ the time to perform the segmentation based on Bayesian theory $\left(t_{b} \approx 10 \mathrm{~min}\right)$. Approximately, $t_{\text {probabilistic }} \approx 30 \mathrm{~min}$.

\section{Discussion}

In this work, the atlas-based methodology has been studied to perform the complete delineation of the pectoral muscle in breast MRI, which has not been done previously. Fully automatic and dedicated multi-atlas and probabilistic frameworks have been proposed and tested on 27 different patients.

The obtained results are satisfactory in both frameworks, with DSC values higher than the computed inter-observer variability. It proves the high reliability of atlas-based segmentation methods to perform pectoral delineations. However, we are aware that the evaluation and the construction of the atlases were performed with annotations from a single viewer, as obtaining 3-dimensional manual segmentations is a time consuming task. The low inter-observer DSC value explains the difficulty and subjectivity to delineate the pectoral muscle. Its shape has high-variability and cartilage, intercostal muscles and fatty tissue also appear in the area. The inclusion of these tissues depends on the observer opinion.

As it was expected, multi-atlas segmentation appears to be more consistent than probabilistic. This is explained by the fact that multi-atlas approach includes an atlas selection step to choose the most similar atlas compared to the 
segmented volume. In the probabilistic framework, when the target differs considerably from the reference atlas and the registration can not compensate the differences, the final segmentation becomes affected with slightly poorer results. However, the computation time for a multi-atlas segmentation is 14 times larger.

Considering the influence of atlas selection, in future work we will study a multi-probabilistic atlas framework. A larger dataset will be created with annotations from different observers. We will group different breast MRI populations based on shape. For each population, a probabilistic atlas will be built. The most similar atlas to the image at hand will be chosen for segmentation.

\section{References}

1. Aljabar, P., Heckemann, R., Hammers, A., Hajnal, J., Rueckert, D.: Multi-atlas based segmentation of brain images: Atlas selection and its effect on accuracy. NeuroImage 46(3), 726-738 (2009)

2. Boyd, N., Guo, H., Martin, L., Sun, L., Stone, J., Fishell, E., Jong, A., Hislop, G., Chiarelli, A., Minkin, S., Yaffe, M.: Mammographic density and the risk and detection of breast cancer. N. Engl. J. Med. 356, 227-236 (2007)

3. Camilus, K.S., Govindan, V.K., Sathidevi, P.S.: Computer-aided identification of the pectoral muscle in digitized mammograms. Journal of Digital Imaging 23, 562$580(2010)$

4. Gallego, C., Martel, A.L.: Automatic model-based 3d segmentation of the breast in mri. In: Proc. of SPIE 2011, vol. 7962 (2011)

5. Gubern-Mérida, A., Kallenberg, M., Martí, R., Karssemeijer, N.: Fully automatic fibroglandular tissue segmentation in breast mri: an atlas-based approach. In: MICCAI 2011: Workshop on Breast Image Analysis pp. 73-80 (2011)

6. Klein, S., van der Heide, U., Lips, I., van Vulpen, M., Staring, M., Pluim, J.P.W.: Automatic segmentation of the prostate in $3 \mathrm{~d} \mathrm{mr}$ images by atlas matching using localized mutual information. Medical Physics 35(4), 1407-1417 (2008)

7. Klein, S., Staring, M., Murphy, K., Viergever, M., Pluim, J.: Elastix: a toolbox for intensity based medical image registration. IEEE Transactions on Medical Imaging 29(1), 196-205 (2010)

8. Klifa, C., Carballido-Gamio, J., Wilmes, L., Laprie, A., Shepherd, J., Gibbs, J., Fan, B., Noworolski, S., Hylton, N.: Magnetic resonance imaging for secondary assessment of breast density in a high-risk cohort. Magnetic Resonance Imaging 28(1), 8-15 (2009)

9. Nie, K., Chang, D., Chen, J., Shih, T., Hsu, C., Nalcioglu, O., Su, M.: Impact of skin removal on quantitative measurement of breast density using mri. Med. Phys. 37(1), 227-233 (2010)

10. Wang, L., Filippatos, K., Friman, O., Hahn, H.K.: Fully automated segmentation of the pectoralis muscle boundary in breast mr images. In: Proc. of SPIE 2011, vol. 7963 (2011)

11. Yankeelov, T.E., Luci, J.J., Lepage, M., Li, R., Debusk, L., Lin, P.C., Price, R.R., Gore, J.C.: Quantitative pharmacokinetic analysis of dce-mri data without an arterial input function: a reference region model. Magn. Reson. Imaging 23(4), 519-529 (2005) 